\title{
A thermodynamic solution model for Li-free tourmaline
}

RoOzen, S. ${ }^{{ }^{*}}$, van Hinsberg, V.J. ${ }^{1}$, DAChs, E. ${ }^{2}$, BENISEK, A. ${ }^{2}$, GREVEL K.D. ${ }^{3}$, RYAN D.H. ${ }^{4}$ AND BUD'KO, S. ${ }^{5}$

${ }^{1}$ Dept. Earth and Planetary Sciences, McGill University, Montreal, Canada. (*correspondence: stanroozen@gmail.com)

${ }^{2}$ Dept. Chemistry and Physics of Materials, University of Salzburg, Salzburg, Austria.

${ }^{3}$ Institut für Geowissenschaften, Bereich Mineralogie, Friedrich-Schiller-Universität Jena, Jena, Germany.

${ }^{4}$ Physics Dept., McGill University, Montreal, Canada.

${ }^{5}$ Department of Physics and Astronomy, Iowa State University, Ames, USA.

Tourmaline can adjust its composition to suit a wide variety of environments, and therefore displays a remarkable stability range in terms of pressure, temperature, fluid, and host-rock composition [1]. Its composition is diagnostic of its growth setting and therefore presents a record of a rocks geochemical history. To unlock this record, we thoroughly need to understand its phase relationships. At present, the only thermodynamic model for tourmaline is built on estimated properties [2], which allows for semi-quantitative modeling only. Here we present new thermodynamic measurements that form the foundation for a thermodynamic solution model for the Li-free tourmaline system. Our sample set consists of synthesized end-members and natural intermediate solid solutions of tourmaline in the dravite-schorl-olenite-uviteferuvite-magnesiofoitite-foitite system. We characterized all samples in detail using WDS-EPMA for major elements, LAICP-MS for trace elements, Mössbauer for Fe-valence, single crystal-XRD for long-range, and Raman for short-range order. We measured low-temperature heat capacities (Cp) by relaxation calorimetry and extracted third-law and excess entropies from these data. Tourmaline solubilities from our synthesis experiments constrain enthalpy. We obtained hightemperature Cp by DSC, which allows us to extrapolate our standard state properties to higher temperatures. We calculated molar volumes from the Rietveld refinement of our single crystal-XRD data. Finally, we combined the measured thermodynamic properties with literature data to constrain an internally consistent thermodynamic parameter set for tourmaline, which is integrated into common thermodynamic databases to permit modeling of tourmaline stability in igneous, metamorphic, or hydrothermal systems.

[1] van Hinsberg et al. (2011) Can. Mineral. 49, 1-16. [2] van Hinsberg \& Schumacher (2007) J. Metamorph. Geol. 25, 769-779. 